\title{
Seroprevalencia contra agentes ToRCH en mujeres indígenas en edad fértil, estado Zulia, Venezuela
}

\author{
Francisca Mercedes Monsalve-Castillo, Luciana Ana Costa-León¹, María Elena Castellano', \\ Anais Suárez ${ }^{1}$, Ricardo José Atencio² \\ ${ }^{1}$ Cátedra de Virología, Escuela de Bioanálisis, Facultad de Medicina, Universidad del Zulia, Maracaibo, Estado \\ Zulia, Venezuela \\ ${ }^{2}$ Laboratorio Regional de Referencia Virológica, Facultad de Medicina, Universidad del Zulia, Maracaibo, Estado \\ Zulia, Venezuela
}

Introducción. El termino ToRCH comprende a los patógenos Toxoplasma gondii, virus de la rubéola, citomegalovirus y virus herpes simple 1 y 2 . En mujeres embarazadas expuestas pueden ser causa de abortos y malformaciones congénitas en el neonato.

Objetivo. Determinar la seroprevalencia de infección por los agentes causantes del síndrome ToRCH en mujeres en edad fértil de algunas comunidades indígenas yukpa de Venezuela.

Materiales y métodos. En el año 2007 fueron seleccionadas 109 muestras de 151 mujeres, en edades comprendidas entre 14 y 40 años. La detección de anticuerpos se hizo por el método de inmunoensayo enzimático indirecto o ELISA de Smartest Diagnostics ${ }^{\text {TM }}$.

Resultados. El 85,5\% presentó anticuerpos contra T. gondii, el 95,4 \% para rubéola, el 75,2 \% para citomegalovirus y el $97,2 \%$ para el virus herpes simple 1 y 2 . Se observa que el $21,1 \%$ y el 30,2 $\%$ presentaron relación entre la variable aborto y las infecciones por citomegalovirus y virus herpes simple 1 y 2 , respectivamente.

Conclusiones. Existe alta seroprevalencia de infecciones por los agentes causantes del síndrome $\mathrm{ToRCH}$ en mujeres en edad fértil de la etnia indígena yukpa. Las condiciones sanitarias precarias y el consumo de agua contaminada con ooquistes, favorecen la adquisición de la infección por $T$. gondii. El hacinamiento, el inicio a temprana de edad de la actividad sexual y el número de parejas, pueden incidir en la presencia de citomegalovirus y virus herpes simple 1 y 2.

Palabras clave: Toxoplasma, rubéola, citomegalovirus, herpesvirus humano 1, herpesvirus humano 2 , estudios seroepidemiológicos, mujer, periodo fértil, población indígena, epidemiología.

doi: http://dx.doi.org/10.7705/biomedica.v32i4.749

\section{Prevalence of infectious agents in indigenous women of childbearing age in Venezuela}

Introduction. The $\mathrm{ToRCH}$ syndrome includes the following infectious pathogens: Toxoplasma gondii, rubella, cytomegalovirus and herpes simplex virus 1 and 2. In susceptible pregnant women, these pathogens can cause abortions and congenital malformation in the newborn babies.

Objective. The seroprevalence of infection by $\mathrm{ToRCH}$ agents was determined in women of childbearing age in several Venezuelan Yukpa indigenous communities.

Material and methods. In 2007, 109 samples were selected from 151 women with an age range of 14 to 40 years old. The determination of antibodies against $\mathrm{ToRCH}$ agents was carried out through the indirect enzyme immunoassay technique by ELISA's technique of Smartest Diagnostics.

Results. Of the 109 samples, 85.5\% presented antibodies against T. gondii, $95.4 \%$ for rubella, $75.2 \%$ for cytomegalovirus and $97.2 \%$ for and herpes simplex virus 1 and 2 . A relationship between abortion and infection by cytomegalovirus and herpes simplex virus 1 and 2 was noted in $21.1 \%$ and $30.2 \%$ of women presented, respectively.

Conclusions. The findings show a high prevalence of $\mathrm{ToRCH}$ agents in women in childbearing age in Yukpa indigenous communities in Venezuela. Poor sanitary conditions and consumption of water contaminated with oocysts may be an important way of transmission of $T$. gondii. Overcrowding in the communities, sexual activity at an early age and number of partners and may be related to the presence of cytomegalovirus and herpes simplex virus HSV-1 and 2.

\section{Contribución de los autores:}

Francisca M. Monsalve-Castillo: concepción y diseño del trabajo, y redacción del manuscrito. Luciana Costa-León: asesoría técnica.

Francisca M. Monsalve-Castillo, Luciana Costa-León y Anais Suárez: analizaron e interpretaron los resultados.

María Elena Castellano, Anais Suárez y Ricardo Atencio: recolectaron y obtuvieron los datos.

Todos los autores participaron en la revisión crítica y aprobación de la versión final del manuscrito. 
Keywords: Toxoplasma, rubella, cytomegalovirus; herpesvirus 1, human; herpesvirus 2, human; seroepidemiologic studies, women, fertile period, indigenous population, epidemiology.

doi: http://dx.doi.org/10.7705/biomedica.v32i4.749

El término ToRCH es el acrónimo bajo el cual se agrupa una serie de microorganismos patógenos causantes de infecciones intrauterinas, como son: Toxoplasma gondii, virus de la rubéola, citomegalovirus (CMV) y los virus herpes simple 1 y 2 (VHS-1 y 2), que presentan similitudes y diferencias en sus mecanismos de transmisión y aspectos epidemiológicos (1).

Las secuelas en mujeres gestantes expuestas suelen causar serias manifestaciones en el producto, que van desde un aborto espontáneo hasta malformaciones congénitas, dependiendo de la edad de gestación en la cual ocurre la primoinfección (2).

La clásica tríada asociada a la infección congénita por T. gondii incluye; hidrocefalia, calcificaciones intracraneales y lesiones oculares. La infección por virus de la rubéola causa defectos al nacer, como pérdida de la visión, de la audición y retardo mental, conocido como el síndrome de rubéola congénita. El citomegalovirus presenta similares manifestaciones a las de la toxoplasmosis y la rubéola. Mientras que la infección neonatal por los virus herpes simple 1 y 2 se manifiesta por erupciones en piel, ojos y boca, diseminándose a otros órganos (hígado, bazo) y el sistema nervioso central, la mayoría de estas manifestaciones son causadas por el VHS-2 (3).

La prevalencia de estos agentes varía en los diferentes ámbitos geográficos. Para $T$. gondii, se informa una prevalencia que oscila entre 7,4 y 64,9 $\%$ en países occidentales (4-8). En Venezuela, se ubica entre 36,6 y $49,8 \%(9,10)$ en la población general y, en comunidades rurales e indígenas, en $49,7 \%(11)$.

La inmunidad para rubéola a nivel mundial es alta, gracias a los programas de vacunación implementados desde 1969 y cuya eficacia es del $95 \%$, con un 92,5 a $99 \%$ en la mayoría de los países (12-16). En el 2005, Venezuela presentó una disminución en el número de casos (17); sin

\section{Correspondencia:}

Francisca Monsalve-Castillo, Calle $67^{a}$ con Avenida $14^{a}$, Edificio Orosi, apartamento 4-B, Maracaibo, Venezuela

Teléfono-fax: (58-261) 7529286

monsalve22000@gmail.com

Recibido: 30/08/11; aceptado:02/06/12 embargo, no se tienen datos precisos sobre la situación actual.

La infección por CMV es común entre mujeres en edad fértil, su prevalencia oscila entre 45 y 100 \% (18-21). En Venezuela para el año 2002, la prevalencia se ubicó en 93,3\% (22); mientras que para el VHS-1 se encontraba entre 52,4 y $100 \%$ y, entre 4 y $30 \%$, para el VHS-2 $(23,24)$.

Las comunidades indígenas yukpa que habitan en la Sierra de Perijá, estado Zulia en Venezuela, se caracterizan por presentar condiciones sanitarias precarias, bajo nivel socioeconómico y educativo, y una deficiente atención médica, lo que favorece la adquisición de infecciones virales y parasitarias (25-28).

La mayoría de las investigaciones sobre los agentes ToRCH se ha llevado a cabo en mujeres gestantes e infecciones congénitas; es poca la información obtenida en mujeres en edad fértil, lo que aportaría al conocimiento previo sobre la prevalencia y propensión a estas infecciones.

El objetivo del presente estudio fue determinar la seroprevalencia de las infecciones causadas por los agentes del síndrome ToRCH en mujeres en edad fértil de algunas comunidades indígenas yukpa de Venezuela, e identificar el comportamiento epidemiológico de estos agentes y los factores de riesgo involucrados en su diseminación.

\section{Materiales y métodos}

\section{Ámbito geográfico}

La población yukpa estudiada habita en la Sierra de Perijá, estado Zulia, ubicada en la cordillera de los Andes, que conforma el límite occidental de la cuenca hidrográfica del Lago de Maracaibo, en la zona fronteriza entre Colombia y Venezuela, zona caracterizada por un sistema natural que combina una rica diversidad de biomas. Las precipitaciones son abundantes $(1.000-2.000 \mathrm{~mm})$, con temperatura máxima de $30^{\circ} \mathrm{C}$ y mínima de $13^{\circ} \mathrm{C}$.

\section{Población}

En noviembre del 2007 se tomaron muestras sanguíneas a un universo de 151 mujeres, y se seleccionaron 109 mujeres en edad fértil (1440 años), pertenecientes a cuatro comunidades indígenas yukpa: $40 / 54$ de la comunidad del 
Tokuko, 33/46 de la comunidad de Kasmera, 21/31 en Yasa y 15/20 en Neremú.

Estas comunidades se caracterizan por ser de naturaleza endogámica, de tipo matriarcal, y su organización social se rige por caciques o cabildos. Su actividad sexual comienza a temprana edad. Al conseguir pareja, los hijos hombres y mujeres siguen conviviendo con la madre, y hasta 10 personas llegan a habitar en un solo ambiente: la vivienda. Su aseo y necesidades personales las llevan a cabo a campo abierto cerca de la vivienda. Se observa la convivencia con animales y el agua para consumo es obtenida del río más cercano a cada comunidad.

Los criterios de inclusión fueron: sexo femenino, pertenecer al grupo en edad fértil (14-40 años) y ser nativa de la comunidad yukpa.

Previo consentimiento, se obtuvo la información demográfica, clínica y de factores de riesgo de cada mujer, mediante un instrumento de recolección de datos (edad, número de parejas, aborto, número de abortos). En el presente estudio, las limitaciones fueron la diferencia de idiomas (español-yukpa) y, en la mayoría de las mujeres, el desconocimiento de su edad y la falta de memoria para recordar el año de nacimiento.

\section{Muestra y métodos serológicos}

A cada mujer se le tomaron $5 \mathrm{ml}$ de sangre por punción venosa en tubo de Vacutainer ${ }^{\circledR}$, sin anticoagulante, la cual fue centrifugada a 1.000 rpm para obtener el suero. Las muestras de suero fueron guardadas en alícuotas de $500 \mu \mathrm{l}$ para ser transportadas en hielo seco, desde la comunidad hasta el Laboratorio Regional de Referencias Virológicas de la Facultad de Medicina, donde fueron almacenadas a $-20^{\circ} \mathrm{C}$ hasta el momento de su procesamiento.

Las determinaciones de inmunoglobulinas de la clase IgG anti-CMV, anti- toxoplasma, anti-rubéola y anti-VHS-1 y 2, se realizaron por el método de inmunoensayo enzimático indirecto o ELISA de Smartest Diagnostics ${ }^{T M}$, Israel, el cual tiene una sensibilidad y una especificidad mayor del $98 \%$ para cada uno de los agentes evaluados.

\section{Análisis estadístico}

Esta investigación fue de tipo descriptiva, transeccional, de inferencias, no experimental. Los resultados obtenidos se expresaron en valores absolutos y porcentajes. Se ejecutó la prueba de ji al cuadrado, mediante el programa SPSS $15.0^{\text {TM }}$ para Windows, tomando un $95 \%$ como índice de confiabilidad y toda probabilidad (p) menor de 0,05 se consideró significativa. Las variables evaluadas (edad, número de parejas, aborto, número de abortos) se presentan en tablas de distribución de frecuencia.

\section{Consideraciones éticas}

El proyecto contó con la aprobación del Consejo de Desarrollo Científico y Humanístico de la Universidad del Zulia, en oficio de fecha 19 de marzo de 2009 bajo el número CC 0126-08, cumpliendo con las normativas establecidas para estudios en humanos de acuerdo con la Declaración de Helsinki de 2008. Para el presente estudio se solicitó y obtuvo el consentimiento informado de las mujeres estudiadas, a las cuales se les hizo entrega posterior de los resultados obtenidos.

\section{Resultados}

Se evaluaron el $74 \%, 71,7 \%, 67,7 \%$ y $75 \%$ de las mujeres en edad fértil de las comunidades del Tokuko, Kasmera, Yasa y Neremú, respectivamente. Los resultados indican que de las 109 mujeres en edad fértil evaluadas, el $85,5 \%$ presentaba anticuerpos contra T. gondii, el 95,4\%, para rubéola, el 75,2 \%, para CMV y el 97,2\%, para VHS-1 y 2, sin diferencias significativas entre comunidades (cuadro 1).

La media de las edades fue de 26,6 $\pm 7,2$ años. Los grupos etarios fueron clasificados entre 14 y 19 años, 20 y 25 años, 26 y 31 años y mayores de 32 años. Se observó un incremento en la prevalencia de anticuerpos contra $T$. gondii de acuerdo con la edad, de $60 \%$ a $100 \%$ en las comunidades de Kasmera, Yasa y Tokuko. En Neremú, todas las mujeres estudiadas tenían anticuerpos contra $T$ gondii, excepto en el grupo etario de 20 a 25 años, que presentó una prevalencia de $66,6 \%$.

En relación con la rubéola, todos los grupos etarios de las comunidades de Yasa y Neremú eran inmunes al virus. En el Tokuko, a excepción del grupo etario de 26 a 31 años que presentó una prevalencia de $88,8 \%$, el resto presentaba inmunidad. En Kasmera, se observaron prevalencias de $60 \%$, $72,7 \%, 37,5 \%$ y $88,9 \%$, en las edades de 14 a 19 años, 20 a 25 años, 26 a 31 años y mayores de 32 años, respectivamente.

La mayor inmunidad (100\%) para el CMV se observó en el grupo etario de 14 a 19 años en las comunidades de Tokuko, Kasmera y Neremú. En la comunidad de Yasa la prevalencia se incrementó 
Cuadro 1. Prevalencia de anti-ToRCH en mujeres en edad fértil de comunidades indígenas yukpa, estado Zulia, Venezuela

\begin{tabular}{|c|c|c|c|c|c|c|c|c|c|}
\hline & & \multicolumn{2}{|c|}{ Anti-toxo lgG } & \multicolumn{2}{|c|}{ Anti-rubéola IgG } & \multicolumn{2}{|c|}{ Anti-CMV IgG } & \multicolumn{2}{|c|}{ Anti-VHS-1 y $2 \lg G$} \\
\hline & & Positivo & $\%$ & Positivo & $\%$ & Positivo & $\%$ & Positivo & $\%$ \\
\hline \multicolumn{10}{|c|}{ Comunidad } \\
\hline Tokuko & $(n=40)$ & 35 & 87,5 & 38 & 95,0 & 34 & 85,0 & 40 & 100 \\
\hline Kasmera & $(n=33)$ & 25 & 75,7 & 30 & 91,0 & 24 & 72,7 & 31 & 94,0 \\
\hline Yasa & $(n=21)$ & 18 & 85,7 & 21 & 100 & 11 & 52,3 & 20 & 95,2 \\
\hline Neremú & $(n=15)$ & 12 & 80,0 & 15 & 100 & 13 & 86,6 & 15 & 100 \\
\hline Total & $n=109$ & 90 & 85,5 & 104 & 95,4 & 82 & 75,2 & 106 & 97,2 \\
\hline
\end{tabular}

$\mathrm{n}$ : número de mujeres

Cuadro 2. Asociación entre factores de riesgo demográficos y prevalencia en mujeres en edad fértil de comunidades indígenas yukpa, estado Zulia, Venezuela

\begin{tabular}{|c|c|c|c|c|c|c|c|c|c|c|c|c|c|}
\hline & № de parejas & $\mathbf{F}$ & $\%$ & $\mathbf{F}$ & $\%$ & $\mathbf{F}$ & $\%$ & $\mathbf{F}$ & $\%$ & $\mathbf{F}$ & $\%$ & $\mathbf{P}$ & IC $95 \%$ \\
\hline \multirow[t]{2}{*}{$\mathrm{CMV}+$} & 1 & 10 & 25,0 & 6 & $(18,1)$ & 2 & 9,5 & 5 & 33,3 & 23 & $21,1\}$ & 0,07 & $0,60-1,03$ \\
\hline & $>1$ & 19 & 47,5 & 18 & $(54,5)$ & 8 & 38,1 & 8 & 53,3 & 53 & $48,6]$ & & \\
\hline \multirow[t]{3}{*}{ VHS- 1 y $2+$} & 1 & 12 & 30,0 & 8 & $(24,2)$ & 5 & 23,8 & 5 & 33,3 & 28 & $25,6\}$ & 0,22 & $0,85-1,04$ \\
\hline & $>1$ & 23 & 57,5 & 23 & $(69,7)$ & 15 & 71,4 & 10 & 66,6 & 66 & $60,5]$ & & \\
\hline & Abortos & & & & & & & & & & & & \\
\hline \multirow[t]{2}{*}{$\mathrm{CMV}+$} & No & 28 & 70,0 & 16 & $(48,4)$ & 7 & 33,3 & 8 & 53,3 & 59 & 54,1 & 0,24 & $0,19-0,39$ \\
\hline & Sí & 6 & 15,0 & 4 & $(24,2)$ & 4 & 19,0 & 5 & 33,3 & 23 & $21,1]$ & & \\
\hline \multirow[t]{3}{*}{ VHS-1 y 2+ } & No & 31 & 77,5 & 20 & $(60,6)$ & 13 & 61,9 & 9 & 60,0 & 73 & 66,7 & 0,24 & $0,22-0,40$ \\
\hline & Sí & 9 & 22,5 & 11 & $(33,3)$ & 7 & 33,3 & 6 & 40,0 & 33 & 30,2 & & \\
\hline & № de abortos & & & & & & & & & & & & \\
\hline \multirow[t]{2}{*}{ CMV+ } & 1 & 4 & $(10,0)$ & 5 & $(15,1)$ & 3 & $(14,2)$ & 2 & $(13,3)$ & 14 & $(12,8)\}$ & 0,67 & $0,45-0,88$ \\
\hline & $>1$ & 2 & $(5,0)$ & 3 & $(9,0)$ & 1 & $(4,7)$ & 3 & $(20,0)$ & 9 & $(8,2)]$ & & \\
\hline \multirow[t]{2}{*}{ VHS-1 y 2+ } & 1 & 7 & $(17,5)$ & 7 & $(21,2)$ & 3 & $(14,2)$ & 3 & $(20,0)$ & 20 & $(18,3)\}$ & 0,51 & $0,79-1,03$ \\
\hline & $>1$ & 2 & $(5,0)$ & 4 & $(12,1)$ & 4 & $(19,0)$ & 3 & $(20,0)$ & 13 & $(11,9)]$ & & \\
\hline
\end{tabular}

F: frecuencia; P: probabilidad; IC $95 \%$ : intervalo de confianza de $95 \%$

de $57,7 \%$ a $100 \%$, según el grupo etario. En las comunidades del Tokuko y Neremú, todos los grupos etarios fueron inmunes a los VHS-1 y 2. En Kasmera y Yasa, los porcentajes encontrados aumentaron, de acuerdo con el grupo etario, de $80 \%$ a $100 \%$.

El cuadro 2 muestra aquellos factores de riesgo que hubieron podido tener una relación directa con las infecciones por CMV y VHS-1 y 2. Al evaluar la relación entre el número de parejas y estas infecciones, se indica que el $48,6 \%$ y el $60,5 \%$ que refirieron tener más de una pareja, fueron seropositivas para CMV y VHS-1 y 2.

Se observó que el 21,1 \% y el 30,2 \% presentaron relación entre la variable "aborto" y las infecciones por CMV y VHS 1 y 2, sin llegar a ser significativa. El $8,2 \%$ de las mujeres seropositivas para CMV y el $11,9 \%$ de las positivas para los VHS-1 y 2 , tenían antecedentes de más de un aborto.

\section{Discusión}

El conocimiento de la seroprevalencia y exposición a infecciones por los agentes $\mathrm{ToRCH}$ en mujeres en edad fértil, contribuye a la implementación de medidas de prevención de las complicaciones que suelen causar estos agentes en el feto.

El presente estudio muestra una alta seroprevalencia de infecciones por agentes $\mathrm{ToRCH}$ en mujeres en edad fértil de las comunidades indígenas yukpa estudiadas. La seroprevalencia de infección por T. gondii $(85,5 \%)$ es similar a la encontrada en la etnia guajibos del estado Amazonas ( $88 \%$ ), en Venezuela (29) y en comunidades indígenas en Brasil $(80,4 \%)$ (30). Sin embargo, se encuentra por encima de las señaladas en estudios previos realizados en asentamientos rurales e indígenas de Venezuela y otros países (33 \%-73,5 \%) $(9,10,31,32)$, así como en algunos estudios realizados en mujeres en edad fértil (8,31-34).

La alta seroprevalencia de anticuerpos a T. gondii en estas comunidades no puede ser explicada por la forma más común de transmisión, como es la ingestión de quistes presentes en carnes insuficientemente cocidas, debido a que su dieta es principalmente a base de tubérculos, complementada por la pesca. 
La vía más probable de adquisición de $T$. gondii fue el consumo de agua no tratada, obtenida del río más cercano a cada comunidad, la cual pudo haber estado contaminada con ooquistes eliminados por gatos o felinos que habitan en las cercanías de estos ríos o de los animales que conviven en forma intradomiciliaria con los individuos estudiados. Algunas investigaciones consideran que el consumo de agua no filtrada es un factor importante en la adquisición de esta infección $(10,33,35)$.

Como en investigaciones previas $(9,11,35)$, no se encontró relación entre la prevalencia de la infección y la presencia de animales. Sin embargo, no se descarta su papel en la adquisición de la misma, ya que adultos, y niños principalmente, suelen estar semidesnudos y descalzos, favoreciéndose el contacto con heces de animales domésticos. Esto podría explicar por qué el grupo etario de 14 a 19 años presentaba $60 \%$ a $100 \%$ de inmunidad a T. gondii.

La meta de la Organización Mundial de la Salud fue el control de la rubéola congénita para el año 2010. La cobertura alcanzada durante las campañas ha llegado a más del $95 \%$ en varios países (36). En Venezuela se llevó a cabo en el año 2007, llegando al $90 \%$ de las comunidades indígenas (37).

Para el momento de la toma de la muestra, no se tenía información de que estas comunidades hubieran sido vacunadas durante el mes previo. $\mathrm{La}$ alta seroprevalencia $(95,4 \%)$ encontrada en la población general, hace suponer que la misma puede deberse a anticuerpos de la vacuna. Los anticuerpos IgG detectados no permiten diferenciar entre la inmunidad adquirida naturalmente y la inducida por la vacuna. La estimulación de anticuerpos por vacuna es de 21 a 28 días, manteniéndose hasta por 15 años. La seroprevalencia encontrada apoya lo expresado en varias investigaciones sobre la eficacia de la vacuna en cifras que superan el $95 \%$.

Porcentajes de $5 \%$ y $9 \%$ de exposición al virus de la rubéola, se observaron en las poblaciones de Tokuko y Kasmera; este último fue superior a los encontrados en diversos estudios (12-14). Lo anterior, sumado a la ausencia del síndrome de rubéola congénita en niños de estas comunidades, podría indicar que este virus no ha entrado en las mismas. De ser cierto lo anterior, las características étnicas de estas comunidades pueden tener alguna relevancia en la protección contra la infección por el virus de la rubéola. Los estudios muestran diferencias significativas entre grupos étnicamente diferentes $(38,39)$. La realización de este estudio antes de la vacunación, aclararía estas dudas.

Los factores tales como bajas condiciones socioeconómicas, hacinamiento, inicio de la actividad sexual a temprana edad y número de parejas sexuales, no presentaron asociación alguna con la seroprevalencia de infección por CMV y VHS-1 y 2. Sin embargo, podrían contribuir al riesgo de exposición, tal como lo señalan los estudios en diversas poblaciones $(18,24,40,41)$.

En relación con las infecciones por los VHS-1 y 2, la prevalencia de anticuerpos ( $97,2 \%$ ) está por encima de las informadas en diferentes investigaciones $(31,42,43)$ y son similares a las encontradas por Brazzale en una comunidad indígena en Australia (40). Aunque esta investigación tuvo la limitación de no haber podido diferenciar entre la infección por VHS-1 y VHS-2, el estudio de Monsalve, et al., en el 2001, señaló una prevalencia de la infección por el VHS-2 de 53,1\% (25). Considerando que la infección por el VHS-1 supera a los porcentajes señalados para el VHS-2, se infiere que estos virus entraron hace muchos años y continúan diseminándose en estas poblaciones.

La alta seroprevalencia de anticuerpos contra CMV y VHS-1 y 2 en estas comunidades, podría explicarse por la similitud en su mecanismo de transmisión y diseminación por fluidos corporales, que incluyen secreciones de mucosas orales y genitales.

La exposición $(24,8 \%)$ a la infección por CMV encontrada en la comunidad de Yasa, podría ser consecuencia del aislamiento geográfico que presenta este asentamiento. Las características étnicas propias de este grupo podrían considerarse como un posible mecanismo de protección ante la infección.

Además, se informa un alto porcentaje de abortos $(21,1 \%$ y $30,2 \%)$ relacionados con las infecciones por CMV y VHS en la población en general, así como un porcentaje importante de abortos a repetición (8,2 \%-11,9\%) para CMV y los VHS-1 y 2 , respectivamente. La presencia de ADN-CMV ha sido detectada en tejido placentario obtenido de abortos espontáneos (44-47). Según Nigro, et al., el CMV es uno de los virus más frecuentemente involucrados en este suceso, el cual durante el embarazo puede atravesar la placenta por viremia o ascender a la misma desde el cérvix, generalmente por reactivación. Otro de los virus implicados es el 
VHS-2, el cual causa infecciones recurrentes del aparato reproductivo, involucrando la placenta y el feto (48).

Del presente estudio se concluye que existe una alta prevalencia de los agentes $\mathrm{ToRCH}$ en mujeres en edad fértil de la etnia indígena yukpa. Las precarias condiciones sanitarias (contacto directo con tierra, convivencia con animales, consumo de agua no potable) favorecen la adquisición de la infección por T. gondii. El hacinamiento, el inicio a temprana de edad de la actividad sexual y el número de parejas, pueden incidir en la presencia de CMV y de VHS-1 y 2, en esta población. Teniendo en cuenta que la transmisión intrauterina está íntimamente relacionada con la inmunidad materna, se requiere el establecimiento de acciones a través de programas de control prenatal. En relación con la rubéola, amerita continuar con el programa de vacunación hasta cubrir toda la población para evitar la adquisición de la infección.

\section{Conflicto de intereses}

Los autores declaran no tener ningún conflicto de interés.

\section{Financiación}

La presente investigación fue subvencionada por el Consejo de Desarrollo Científico y Humanístico de la Universidad del Zulia (CONDES). Subvención № CC 0126-08.

\section{Referencias}

1. Franca CM, Mugayar LR. Intrauterine infections: A literature review. Spec Care Dentist. 2004;24:250-3. http://dx.doi. org/10.1111/j.1754-4505.2004.tb01701.x

2. Ocak S, Zeteroglu S, Ozer C, Dolapcioglu K, Gungoren A. Seroprevalence of Toxoplasma gondii, rubella and cytomegalovirus among pregnant women in southern Turkey. Scand J Infect Dis. 2007;39:231-4. http://dx.doi. org/10.1080/00365540600978880

3. Abu-Madi MA, Behnke JM, Dabritz HA. Toxoplasma gondii seropositivity and co-infection with TORCH pathogens in high-risk patients from Qatar. Am J Trop Med Hyg. 2010;82:626-33. http://dx.doi.org/10.4269/ajtmh.2010.090530

4. Alvarado-Esquivel C, Mercado-Suárez MF, RodríguezBriones A, Fallad-Torres L, Ayala-Ayala JO, NevarezPiedra LJ, et al. Seroepidemiology of infection with Toxoplasma gondii in healthy blood donors of Durango, Mexico. BMC Infect Dis. 2007;7:75. http://dx.doi. org/10.1186/1471-2334-7-75

5. Bartolomé J, Martínez M, Moreno L, Lorente S, Crespo MD. Prevalence and incidence in Albacete, Spain, of Toxoplasma gondii infection in women of childbearing age: Differences between immigrant and non-immigrant (20012007). Rev Esp Salud Pública. 2008;82:333-42.
6. Jones JL, Dargelas V, Roberts J, Press C, Remington JS, Montoya JG. Risk factors for Toxoplasma gondii infection in the United States. Clin Infect Dis. 2009;49:878-84. http:// dx.doi.org/10.1086/605433

7. De Paschale M, Agrappi C, Clerici P, Mirri P, Manco MT, Cavallari S, et al. Seroprevalence and incidence of Toxoplasma gondii infection in the Legnano area of Italy. Clin Microbiol Infect. 2008;14:186-9. http://dx.doi. org/10.1111/j.1469-0691.2007.01883.x

8. Fernandes GC, Azevedo RS, Amaku M, Yu AL, Massad E. Seroepidemiology of Toxoplasma infection in a metropolitan region of Brazil. Epidemiol Infect. 2009;137:1809-15. http:// dx.doi.org/10.1017/S0950268809002799

9. Díaz-Suárez $\mathbf{O}$, Parra $\mathbf{A M}$, Araujo-Fernández $\mathbf{M}$. Seroepidemiology of toxoplasmosis in a marginal community of the municipality of Maracaibo, Zulia State, Venezuela. Invest Clin. 2001;42:107-21.

10. Chacín-Bonilla L, Sánchez-Chávez Y, Estévez J, Larreal I, Molero E. Prevalence of human toxoplasmosis in San Carlos Island, Venezuela. INCI. 2003;28:457-62.

11. Chacín-Bonilla L, Sánchez-Chávez Y, Monsalve F, Estévez J. Seroepidemiology of toxoplasmosis in amerindians from western Venezuela. Am J Trop Med Hyg. $2001 ; 65: 131-5$.

12. Aksakal FN, Maral I, Cirak MY, Aygun R. Rubella seroprevalence among women of childbearing age residing in a rural region: Is there a need for rubella vaccination in Turkey? Jpn J Infect Dis. 2007;60:157-60.

13. Domínguez A, Plans P, Espuñes J, Costa J, Torner N, Cardeñosa $\mathbf{N}$, et al. Rubella immune status of indigenous and immigrant pregnant women in Catalonia, Spain. Eur J Public Health. 2007;17:560-4. http://dx.doi.org/10.1093/ eurpub/ckm034

14. Pedranti MS, Adamo MP, Macedo R, Zapata MT. Prevalence of anti-rubella and anti-parvovirus B19 antibodies in pregnant women in the city of Córdoba, and in women of fertile age in the city of Villa Mercedes, province of San Luis. Rev Argent Microbiol. 2007;39:47-50.

15. Sanz JC, García L, Ramírez R, Ramos B, Ordobás M. Evolución del sarampión, rubéola y parotiditis desde la III Encuesta de Serovigilancia de la Comunidad de Madrid 1999-2000 (III ESVCM). Rev Esp Salud Pública. 2009;83:625-37.

16. Lin CC, Yang CY, Shih CT, Chen BH, Huang YL. Rubella seroepidemiology and catch-up immunization among pregnant women in Taiwan: Comparison between women born in Taiwan and immigrants from six countries in Asia. Am J Trop Med Hyg. 2010;82:40-4. http://dx.doi.org/10.4269/ ajtmh.2010.09-0302

17. Zapata L. Prevención y eliminación del síndrome de rubéola congénita. Rev Obstet Ginecol Venez. 2006;66:193-6.

18. De Ory F, Sanz JC, Castañeda R, Ramírez R, León $\mathbf{P}$, Pachón del Amo I. Cytomegalovirus seroepidemiology in the community of Madrid. Rev Esp Salud Pública. 2001;75:55-62.

19. Staras S, Dollard S, Radford K, Flanders W, Pass R, Cannon M. Seroprevalence of cytomegalovirus infection in the United States, 1988-1994. Clin Infect Dis. 2006;43:1152-3. http://dx.doi.org/10.1086/508173 
20. Adjei A, Armah H, Gbagbo F, Boamah I, Adu C, Asare I. Seroprevalence of HHV-8, CMV, and EBV among the general population in Ghana, West Africa. BMC Infect Dis. 2008;8:111. http://dx.doi.org/10.1186/1471-2334-8-111

21. Cannon MJ, Schmid DS, Hyde TB. Review of cytomegalovirus seroprevalence and demographic characteristics associated with infection. Rev Med Virol. 2010;20:202-13. http://dx.doi.org/10.1002/rmv.655

22. Chacón MR, Naveda O, Castillo O, Flores ME, Casanova L, Castro L, et al. Prevalencia de anticuerpos anticitomegalovirus y anti-virus Epstein-Barr en Valencia, Estado Carabobo, Venezuela. Rev Soc Ven Microbiol. 2002;22:131-5.

23. Pebody RG, Andrews N, Brown D, Gopal R, De Melker $\mathbf{H}$, François $\mathbf{G}$, et al. The seroepidemiology of herpes simplex virus type 1 and 2 in Europe. Sex Transm Infect. 2004;80:185-91. http://dx.doi.org/10.1136/sti.2003.005850

24. Haddow LJ, Sullivan EA, Taylor J, Abel M, Cunningham AL, Tabrizi S, et al. Herpes simplex virus type 2 (HSV-2) infections in women attending an antenatal clinic in the South Pacific island nation of Vanuatu. Sex Transm Dis. 2007;34:258-61. http://dx.doi.org/10.1097/01.olq.0000237774. 29010.30

25. Monsalve F, Estévez J, Costa L, Salas M, Hernández M, Olaya $\mathrm{J}$, et al. Seroepidemiology of herpes simplex virus type 2 in the Amerindian Yukpa population of Zulia state, Venezuela. Rev Med Chile. 2001;129:247-52. http://dx.doi. org/10.4067/S0034-98872001000300002

26. Monsalve-Castillo F, Echevarría JM, Atencio R, Suárez A, Estévez J, Costa-León L, et al. High prevalence of hepatitis B infection in Amerindians in Japreira, Zulia State, Venezuela. Cad Saude Publica. 2008;24:1183-6. http:// dx.doi.org/10.1590/S0102-311X2008000500028

27. Rivero Z, Maldonado A, Bracho Á, Gotera J, Atencio $\mathbf{R}$, Leal $\mathbf{M}$, et al. Enteroparasitosis en indígenas de la comunidad Japrería, estado Zulia, Venezuela. INCI. 2007;32:270-3

28. Maldonado A, Rivero-R Z, Chourio-L G, Díaz I, CalchiLC M, Acurero $\mathbf{H}$, et al. Prevalencia de enteroparásitos y factores ambientales asociados en dos comunidades indígenas del estado Zulia. Kasmera. 2008;36:53-66.

29. De la Rosa M, Bolívar J, Pérez HA. Toxoplasma gondii infection in Amerindians of Venezuelan Amazon. Medicina (B. Aires). 1999;59:759-62.

30. Amendoeira MR, Sobral CA, Teva A, de Lima JN, Klein $\mathrm{CH}$. Serological survey of Toxoplasma gondii infection in isolated Amerindians, Mato Grosso. Rev Soc Bras Med Trop. 2003;36:671-6. http://dx.doi.org/10.1590/S003786822003000600005

31. Vilibic-Cavlek T, Ljubin-Sternak S, Ban M, Kolaric B, Sviben M, Mlinaric-Galinovic G. Seroprevalence of $\mathrm{ToRCH}$ infections in women of childbearing age in Croatia. Matern Fetal Neonatal Med. 2011;24:280-3. http://dx.doi.or $\mathrm{g} / 10.3109 / 14767058.2010 .485233$

32. Neves Bóia M, Carvalho-Costa FA, Campos Sodré F, Trindade Pinto GM, Reis Amendoeira MR. Seroprevalence of Toxoplasma gondii infection among indian people living in lauareté, São Gabriel da Cachoeira, Amazonas, Brazil. Rev Inst Med Trop S Paulo. 2008;50:17-20. http://dx.doi. org/10.1590/S0036-46652008000100004
33. Díaz-Suárez O, Estévez J. Seroepidemiology of toxoplasmosis in women of childbearing age from a marginal community of Maracaibo, Venezuela. Rev Inst Med Trop S Paulo. 2009;51:13-7. http://dx.doi.org/10.1590/S003646652009000100003

34. Bouhamdan SF, Bitar LK, Saghir HJ, Bayan A, Araj GF. Seroprevalence of Toxoplasma antibodies among individuals tested at hospitals and private laboratories in Beirut. J Med Liban. 2010;58:8-11.

35. López-Castillo CA, Díaz-Ramírez J, Gómez-Marín JE. Factores de riesgo en mujeres embarazadas, infectadas por Toxoplasma gondii en Armenia-Colombia. Rev Salud Pública. 2005;7:180-90.

36. Organización Panamericana de la Salud. Acercándonos a la eliminación de la rubéola y síndrome de rubéola congénita en las Américas. 2005;27:1-8. Fecha de consulta: 30 de julio de 2010. Disponible en: http://www.paho.org/ spanish/ad/fch/im/sns2701.pdf

37. Gobierno Bolivariano de Venezuela. Ministerio del Poder Popular para la Comunicación y la Información. Vacunación contra la rubéola en la población indígena ha sido histórica. 2007. Fecha de consulta: 25 de marzo de 2011. Disponible en: www.rnv.gov.ve/noticias

38. Sathanandan D, Gupta L, Liu B, Rutherford A, Lane J. Factors associated with low immunity to rubella infection on antenatalscreening.AustNZJObstetGynaecol.2006;46:172. http://dx.doi.org/10.1111/j.1479-828X.2005.00467.x

39. Mixer RE, Jamrozik K, Newsom D. Ethnicity as a correlate of the uptake of the first dose of mumps, measles and rubella vaccine. J Epidemiol Community Health. 2007;61:797-801. http://dx.doi.org/10.1136/jech.2005.045633

40. Brazzale AG, Russell DB, Cunningham AL, Taylor J, McBride WJ. Seroprevalence of herpes simplex virus type 1 and type 2 among the Indigenous population of Cape York, Far North Queensland, Australia. Sex Health. 2010;7:453-9. http://dx.doi.org/10.1071/SH09098

41. Dowd JB, Zajacova A, Aiello A. Early origins of health disparities: Burden of infection, health, and socioeconomic status in U.S. children. Soc Sci Med. 2009;68:699-707. http://dx.doi.org/10.1016/j.socscimed.2008.12.010

42. Clemens SA, Farhat CK. Seroprevalence of herpes simplex 1-2 antibodies in Brazil. Rev Saude Publica. 2010;44:72634. http://dx.doi.org/10.1590/S0034-89102010000400017

43. Xu F, Sternberg MR, Kottiri BJ, McQuillan GM, Lee FK, Nahmias AJ, et al. Trends in herpes simplex virus type 1 and type 2 seroprevalence in the United States. JAMA. 2006;296:964-73.http://dx.doi.org/10.1001/jama.296. 8.964 .

44. Syridou G, Spanakis N, Konstantinidou A, Piperaki ET, Kafetzis D, Patsouris E, et al. Detection of cytomegalovirus, parvovirus B19 and herpes simplex viruses in cases of intrauterine fetal death: Association with pathological findings. J Med Virol. 2008;80:1776-82. http://dx.doi. org/10.1002/jmv.21293

45. Tamiolakis D, Venizelos I, Lambropoulou M, Kotini A, Barbagadaki S, Nikolaidou S, et al. Human decidual cells activity in women with spontaneous abortions of probable CMV etiology during the first trimester of gestation. An immunohistochemical study with CMV-associated antigen. Acta Medical (Hradec Kralove). 2004;47:195-9. 
46. Nagamori T, Koyano S, Inoue N, Yamada H, Oshima M, Minematsu T, et al. Single cytomegalovirus strain associated with fetal loss and then congenital infection of a subsequent child born to the same mother. J Clin Virol. 2010;49:134-6. http://dx.doi.org/10.1016/j.jcv.2010.06.021

47. Al-Buhtori M, Moore L, Benbow EW, Cooper RJ. Viral detection in hydrops fetalis, spontaneous abortion, and unexplained fetal death in utero. J Med Virol. 2011;3:67984. http://dx.doi.org/10.1002/jmv.22007

48. Nigro G, Mazzocco M, Mattia E, Di Renzo GC, Carta G, Anceschi MM. Role of the infections in recurrent spontaneous abortion. J Matern Fetal Neonatal Med. 2011;24:983-9. http://dx.doi.org/10.3109/14767058.2010.5 47963 